\title{
Best Clustering Around the Color Images
}

\author{
Seyyed Meysam Hosseini , Hasan Farsi , Hadi Sadoghi Yazdi , Member, IACSIT
}

\begin{abstract}
- one of the best way to clustering in color images is to transform R, G, B color space into the target color space by linear transformations that are captured by $3 \times 3$ matrices. The Main target of this paper is introducing new color transform from viewpoint of convex constraint programming. Lip detection is used as benchmark problem for the proposed algorithm. In the New color space, the Lip and non-Lip classes are separated as well. This problem is converted to a convex constraint programming which Genetic Algorithm is used for solving this problem. Founded converting matrix is tested in Lip detection in simple to complex scene. Obtained results over many databases are compared with existing methods which show superiority of the proposed method.
\end{abstract}

Index Terms-Genetic Algorithm, Color space, convex constraint programming, Lip detection, clustering criteria.

\section{INTRODUCTION}

Target color detection is a challenging issue in image processing and machine vision. It is gaining wide portion in recent works in these areas. In this work, generation of a novel multi-objective color space using non-linear convex constraint programming is proposed to deal with target area detection problem in color images. Color is a 3-dimensional psychophysical phenomenon and is represented in color space models whereby individual colors are specified by points in these spaces. R, G, B primaries can produce a gamut of $\left(2^{8}\right)^{3}$ different colors [1]. RGB is the color space usually used in digital images. Each pixels use 8 bits for each one of its color components (R, G and B), in a total of 24 bits for pixel. The color space converter transforms the color information form the RGB space to the other spaces as $\mathrm{YCbCr}$ space and it should maintain the representation of each one of the new space components with the same amount of bits used to represent each component of the input space $(\mathrm{R}, \mathrm{G}$ and $\mathrm{B})$. The calculations performed in the color space conversion from $\mathrm{RGB}$ to $\mathrm{YCbCr}$ are presented below. Parcels of each R, G and B input components are considered in the calculation of the output components in the space $\mathrm{YCbCr}[2]$.

$$
\left[\begin{array}{c}
Y \\
C b \\
C r
\end{array}\right]=\left[\begin{array}{ccc}
0.299 & 0.578 & 0.114 \\
-0.169 & -0.331 & 0.5 \\
0.5 & -0.419 & -0.081
\end{array}\right]\left[\begin{array}{l}
R \\
G \\
B
\end{array}\right]
$$

CIE standardized color order systems by specifying the light source, the observer and the methodology used to derive the values for describing a color. The XYZ color system is also accepted then and it has been used ever since. In this system, Y represents the brightness (or luminance) of the color, while $\mathrm{X}$ and $\mathrm{Z}$ are virtual (or not physically realizable) components of the primary spectra [1]. Many color spaces are presented but other viewpoint is theories of color vision which are derived from the sums and differences of the three cone types. One mechanism (often referred to as the "luminance" mechanism) signals a weighted sum of long-wavelength-selective (L) and medium-wavelength-selective (M) cones, i.e., "L+M" (with some debate regarding the contribution of short wavelength selective (S) cones: [4, 5, and 7]. Two chromatic mechanisms signal weighted sums and differences of the cones. The "L-M" mechanism signals differences between L- and M-cones (and is often referred to as the "red/green" mechanism). The "S-(L+M)" mechanism signals differences between S-cones and the sum of L- and M-cones (and is often referred to as the "blue/yellow" mechanism).

Other viewpoint to color space is selection of best space. In this regard, different transformations of RGB color space (as HSI, HSV and Lab) are compared to find the best method for separating target/clutter or foreground/background and so on in color images taken by a digital photo camera [6]. Some of researchers used a color space in a specific application in the computer vision as skin detection in HSV [7], face recognition in new space based on K-L $\mathrm{L}^{1}$ transform [8], lip tracking into the CIELAB, CIELUV color spaces [9], object recognition into illumination invariant type of RGB color space [10], Image restoration into the CIELAB color space [11], and so on. The performance of an image analysis procedure is known to depend on the choice of the color space. In [12], the properties of six color spaces are discussed for detection of specific surface defects. They show that these defects are well detected when the clustering analysis is performed in the RGB space. Authors of [13] apply two classification methods using different color spaces. By means of a visual appreciation of the results, they also conclude that the RGB space is the best among all the considered color spaces.

This short and non-exhaustive bibliography shows that different authors provide contradictory conclusions about the pertinence of the available color spaces in the context of image analysis. Instead of searching the best classical color space for object detection/classification/segmentation, we propose an original approach based on convex constraint optimization technique for converting of color space which can be used in very application. Linear transformation by $3 \times 3$ matrix is performed and all pixels of target and nontarget are transformed to new space but this matrix must be calculated as in the new space target and non-target are separated as well. Then Lip color detection is used as benchmark problem to test of the proposed algorithm. Color space is mapped to a new space over Lip color data base with Lip and non-Lip color are clustered to best form. For evaluation, clustering criteria is used. Optimum color space in Lip color detection over selected data base is caused Lip

\footnotetext{
${ }^{1}$ Karhunen-Lo`eve
} 
and non-Lip cluster to be separated is created as well. Manifest features of the proposed method involved by Creating of color space from viewpoint of constraint programming, finding optimum color space based on type of application, Effect of the proposed method in a real application.

\section{FOUNDING NEW COLOR SPACE BASED ON CONSTRAINT PROGRAMMING TECHNIQUE}

Many color spaces are related to each other by linear transformations through $3 \times 3$ matrices. Hence a given color, and thereby any color image, can be represented in terms of another color space by transforming its 3-d vector representation using the $3 \times 3$ matrix as follows,

$$
\left[\begin{array}{l}
X_{1} \\
X_{2} \\
X_{3}
\end{array}\right]=\left[\begin{array}{lll}
w_{11} & w_{12} & w_{13} \\
w_{21} & w_{22} & w_{23} \\
w_{31} & w_{32} & w_{33}
\end{array}\right]\left[\begin{array}{l}
R \\
G \\
B
\end{array}\right]
$$

With regard to (4), we can write for each pixel:

$$
\begin{aligned}
& X_{1}=w_{11} R+w_{12} G+w_{13} B \\
& X_{2}=w_{21} R+w_{22} G+w_{23} B \\
& X_{3}=w_{31} R+w_{32} G+w_{33} B
\end{aligned}
$$

$w_{i j}$ in (3) must be founded to create new color space $\left(X_{1}, X_{2}, X_{3}\right)$, in order to optimal clustering vector target and non-target with the following criteria,

$$
\Delta(W)=\frac{\sum_{j=1}^{N} \zeta_{1 j}+\sum_{j=1}^{M} \zeta_{2 j}}{\Gamma}
$$

Where $\zeta_{1 j}$ is the distance of $\mathrm{j}^{\text {th }}$ sample from the center of class 1 , and $\zeta_{2 j}$ is the distance of $\mathrm{j}^{\text {th }}$ sample from the center of class 2 , and $\Gamma$ is the distance between two center class. And $N, M$ are number of sample in class 1,2 respectively and $W$ is a matrix including $w_{i j}$ for $\mathrm{i}, \mathrm{j}=1,2,3$. Now, creating color space problem is converted to an optimization problem which includes some constraints. As said, in this work tow classes determined as Lip and non-Lip classes. In new color space, each pixel is converted using (3) and center of each clusters are obtained using $(5,6)$ as follows,

$$
\left\{\begin{array}{l}
\mu_{X 1}^{1}=w_{11} \bar{R}^{1}+w_{12} \bar{G}^{1}+w_{13} \bar{B}^{1} \\
\mu_{X 2}^{1}=w_{21} \bar{R}^{1}+w_{22} \bar{G}^{1}+w_{23} \bar{B}^{1} \\
\mu_{X 3}^{1}=w_{31} \bar{R}^{1}+w_{32} \bar{G}^{1}+w_{33} \bar{B}^{1}
\end{array}\right.
$$

Where $\mu_{X i}^{k}$ is the center of $\mathrm{k}^{\text {th }}$ cluster in the new color space in the $\mathrm{i}^{\text {th }}$ dimension. $\bar{R}^{i}, \bar{G}^{i}, \bar{B}^{i}$ are mean values of $\mathrm{R}, \mathrm{G}, \mathrm{B}$ components in the $\mathrm{i}^{\text {th }}$ cluster (Lip and non-Lip). $w_{i j}$ are conversion weights. The Distance of each pixel in each cluster to its center and meanwhile the distance between tow classes can be obtained from $(7,8,9)$ :

$$
\begin{aligned}
& d_{i}=\left(w_{11}\left(R_{i}^{1}-\bar{R}\right)+w_{12}\left(G_{i}^{1}-\bar{G}\right)+w_{13}\left(B_{i}^{1}-\bar{B}\right)\right)^{2}+ \\
& \left(w_{21}\left(R_{i}^{1}-\bar{R}\right)+w_{22}\left(G_{i}^{1}-\bar{G}\right)+w_{23}\left(B_{i}^{1}-\bar{B}\right)\right)^{2}+ \\
& \left(w_{31}\left(R_{i}^{1}-\bar{R}\right)+w_{32}\left(G_{i}^{1}-\bar{G}\right)+w_{33}\left(B_{i}^{1}-\bar{B}\right)\right)^{2} \\
& r_{i}=\left(w_{11}\left(R_{i}^{2}-\bar{R}^{2}\right)+w_{12}\left(G_{i}^{2}-\bar{G}^{2}\right)+w_{13}\left(B_{i}^{2}-\bar{B}^{2}\right)\right)^{2}+ \\
& \left(w_{21}\left(R_{i}^{2}-\bar{R}^{2}\right)+w_{22}\left(G_{i}^{2}-\bar{G}^{2}\right)+w_{23}\left(B_{i}^{2}-\bar{B}^{2}\right)\right)^{2}+ \\
& \left(w_{31}\left(R_{i}^{2}-\bar{R}^{2}\right)+w_{32}\left(G_{i}^{2}-\bar{G}^{2}\right)+w_{33}\left(B_{i}^{2}-\bar{B}^{2}\right)\right)^{2} . \\
& \Gamma=\left(w_{11}(\bar{R}-\bar{R})+w_{12}(\bar{G}-\bar{G})+w_{13}(\bar{B}-\bar{B})\right)^{2}+ \\
& \left(w_{21}\left(\frac{1}{R}-\frac{2}{R}\right)+w_{22}(\bar{G}-\bar{G})+w_{23}(\bar{B}-\bar{B})\right)^{2}+ \\
& \left(w_{31}\left(\bar{R}-\bar{R}^{2}\right)+w_{32}(\bar{G}-\bar{G})+w_{33}\left(\bar{B}-\bar{B}^{2}\right)\right)^{2}
\end{aligned}
$$

Where $d_{i}$ and $r_{i}$ are distances of each pixel of Lip and non-Lip from their centers in the new color space respectively. $R_{i}, G_{i}, B_{i}$ are pixel values in RGB space. So, sum of distances for total pixels in each cluster from centers of Lip and non-Lip clusters are obtained by calculating the distance for each pixel in Lip and non-Lip classes. From $(5,6)$ the distance between classes can be obtained to form of (9). Then $\Delta(w)$ is obtained as expressed at Eq.6. Now, this problem converted to one of the most common problems in calculus is that of finding minima (in general, "extrema") of a function subject to constraints. The Genetic Algorithm is a powerful tool for solving this class of problems without the need to explicitly solve the conditions and use them to eliminate extra variables

We follow for minimization of cost function $\Delta(W)$ with following constraints and then In order to minimize clustering error and obtain optimum transformation weights the Genetic Algorithm is utilized. In fact, Genetic Algorithm searches a 9-dimensional space for optimum weights. The key point that validates this method is the generalization capability of the obtained weights. Indeed, because of approximately fixed position of Lip pixels in 3-D space over any color image, weights optimized on some sample image, can lead to acceptable results over any test image.

\section{CONSTRAINTS}

In this paper we are determining several constraint as a bellow that follow of them can guarantee the coverage of 
$\Delta(W)$ into the optimum cost,

$$
\begin{gathered}
1=w_{11}+w_{12}+w_{13} \\
1=w_{21}+w_{22}+w_{23} \\
1=w_{31}+w_{32}+w_{33} \\
256=w_{11} 255+w_{12} 255+w_{13} 255 \\
256=w_{21} 255+w_{22} 255+w_{23} 255 \\
256=w_{31} 255+w_{32} 255+w_{33} 255 \\
R_{i} w_{11}+G_{i} w_{12}+B_{i} w_{13} \leq 256 \\
R_{i} w_{21}+G_{i} w_{22}+B_{i} w_{23} \leq 256 \\
R_{i} w_{31}+G_{i} w_{32}+B_{i} w_{33} \leq 256 \\
\left(R_{i} w_{11}+G_{i} w_{12}+B_{i} w_{13}\right)>0 \\
\left(R_{i} w_{21}+G_{i} w_{22}+B_{i} w_{23}\right)>0 \\
\left(R_{i} w_{31}+G_{i} w_{32}+B_{i} w_{33}\right)>0
\end{gathered}
$$

Also, all weights must be limited into defined range as $a \leq w_{i j} \leq b$.

\section{GENETIC ALGORITHM}

Genetic Algorithms are adaptive heuristic search algorithm premised on the evolutionary ideas of natural selection and genetic. In general genetic algorithm is an iterative procedure that consists of a constant-size population of individuals, each one being represented by a limited string of symbols, known as the genome, encoding a possible solution in a given problem space. This space is known as the search space, consisting of all possible solutions to the problem one is trying to solve. In general, the genetic algorithm is applied to spaces which are too large to be exhaustively searched [16]. The three most important aspects of using genetic algorithms are the definition of the objective function, definition and implementation of the genetic representation, and definition and implementation of the genetic operators. Given below is general algorithm for Genetic algorithms.

\section{GENERAL ALGORITHM FOR GENETIC ALGORITHM}

In general in genetic algorithm there is an initial population which is created from a random selection of solutions. Then fitness value is assigned according to a criteria decided by the person solving the problem. This fitness is assigned to each solution (chromosome) depending on how close it actually is to solving the problem. Those chromosomes with a higher fitness value are more likely to reproduce offspring (which can mutate after reproduction). The offspring is a product of the father and mother, whose composition consists of a combination of genes from them (this process is known as "crossing over". If the new generation contains a solution that produces an output that is close enough or equal to the desired answer then the problem has been solved. If this is not the case, then the new generation will go through the same process as their parents did. One continues this process until a possible solution is reached.
Pseudo code for Genetic Algorithm

\author{
Begin $G A$ \\ $g:=0$ \{ generation counter \} \\ Initialize population $\boldsymbol{P}(\mathrm{g})$ \\ Evaluate population $P(g)$ \{ i.e., compute fitness \\ values $\}$ \\ While not done do \\ $g:=g+1$ \\ Select $P(g)$ from $P(g-1)$ \\ Crossover $P(g)$ \\ Mutate $P(g)$ \\ Evaluate $P(g)$ \\ end while \\ end $G A$
}

Fig.1 Genetic Algorithm

\section{SIMULATION RESULT}

The proposed method is implemented in MATLAB 7 framework. Space conversion weights are obtained using the Lip image that composed by collecting several Lip pixels and these weights are as follows:

$$
W_{i j}=\left[\begin{array}{rrr}
-0.2170 & 1.5337 & -0.7741 \\
-3.2572 & 3.2572 & 0.5428 \\
3.2572 & -3.2572 & 0.5428
\end{array}\right]
$$

Figure 2(a) shows Genetic Algorithm clustering result. But if clustering can be performed in a more sensible space, cost function will be minimized and clustering will be more accurate. As can be seen in figure 2(b), cost function is minimized to -1129 (indicating error of 0.48). Note that in figure 2(b) clustering is illustrated in the original space, and pixels in new space are shown in figure 2(c).Excellence and incredible efficiency of proposed method can be investigated in the generalization capability of the method. Also, one can observe learn and test images in the product space of system to appreciate its ability. 

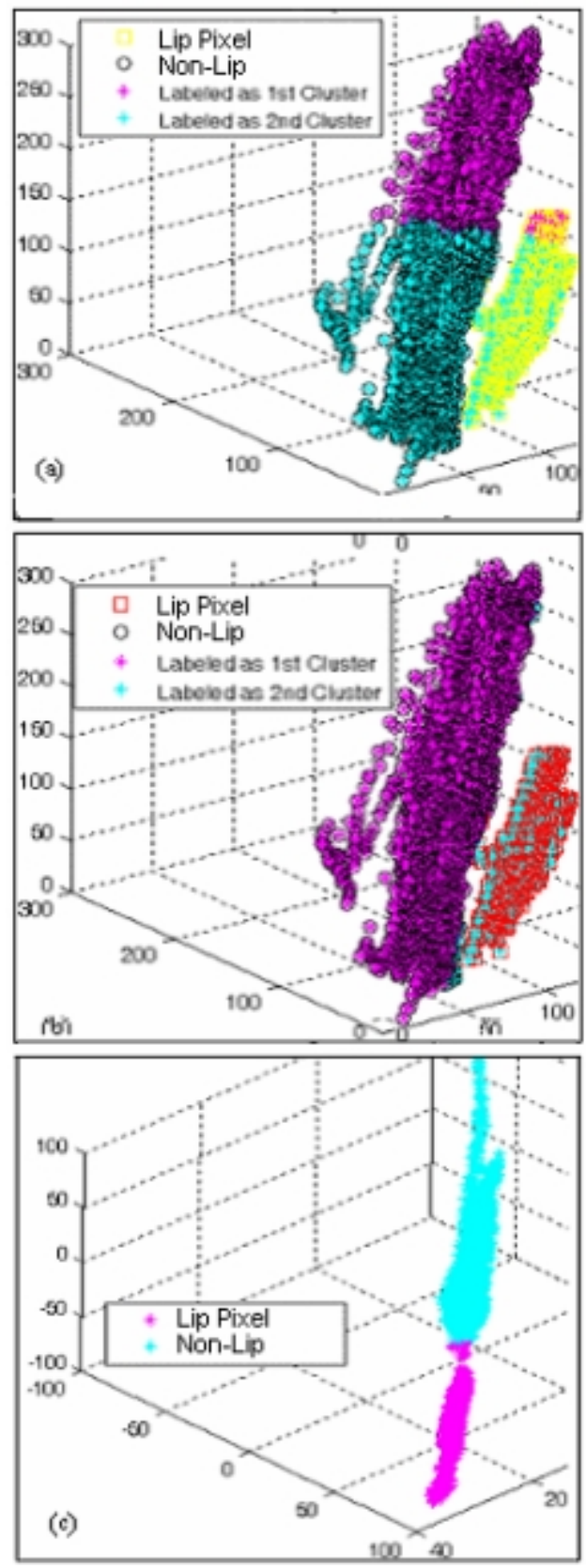

Fig.2 a) Clustering by proposed method b) Lip and non-Lip pixels in original color space c) Clustering in more sensible space.

In Lip detection, due to widely variant lighting condition and presence of Lip-like pixels some defects may arise. This is because of intrinsic defect of pixel-based detection methods and almost is unavoidable. Some other Lip detection methods require several post processing steps to improve their result. Authors of [22] used region-based Lip detector to improve their result. The proposed method shows incredible ability in Lip detection under varying condition of pure to high lighting, indoor and outdoor captured images, and low/high resolution images and also in different databases.

As seen in figure 4, this must be emphasized that just one sample image is used to obtain transformation weights, and then applied on so many test images. As mentioned before, obtained results are comparable and even better than competing methods. As seen in figure 3 Genetic algorithm yields to cost function minimization and obtain optimum transformation weights by repetition and by evolutionary search.

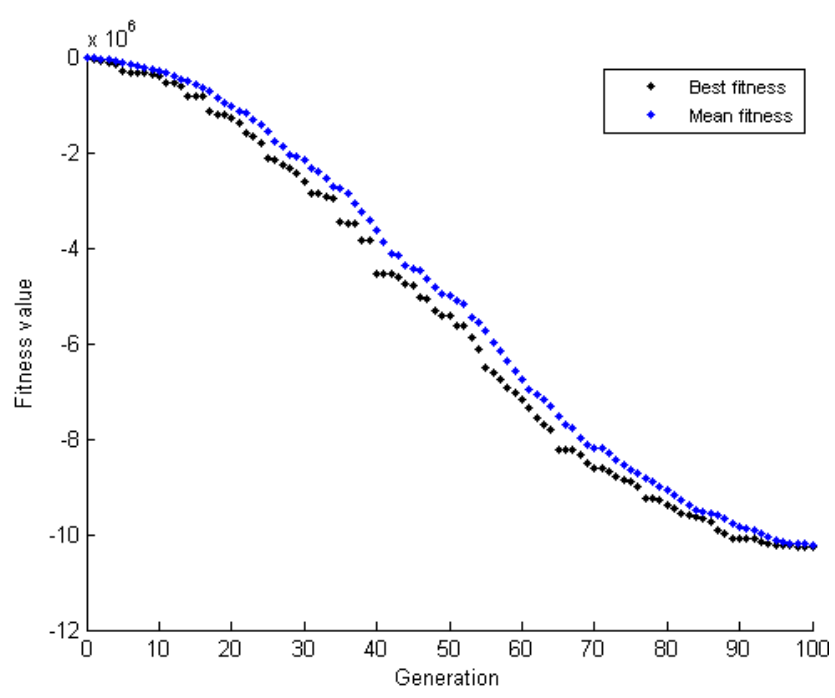

Fig. 3 Genetic Algorithm generation evolution

\section{CONCLUSION}

In this paper, a new idea is presented to solve the frequently applied problem of target color detection. It is based on finding a linear transformation in a way that satisfies a predefined clustering criterion. Thus target detection problem converted into a convex constraint programming problem, which is a famous mathematical problem with definite solution. We presented the method results (for two different target color detection problems) and compared it with newly published works. Also we showed clustering criteria satisfaction visually. A comparative study of color spaces shows the superiority of newly generated space for Lip detection. As far as we know, this method is the most simple and powerful one that has ever been presented. It works under various lighting conditions and for different target color detection problems. Efficiency of this method in other color spaces may be investigated as our future work. Also it is expected that optimization of constraints lead us to better achievements. In other words there is still argument on constraints setup and making them more reliable. We aim to follow these matters in our future works.
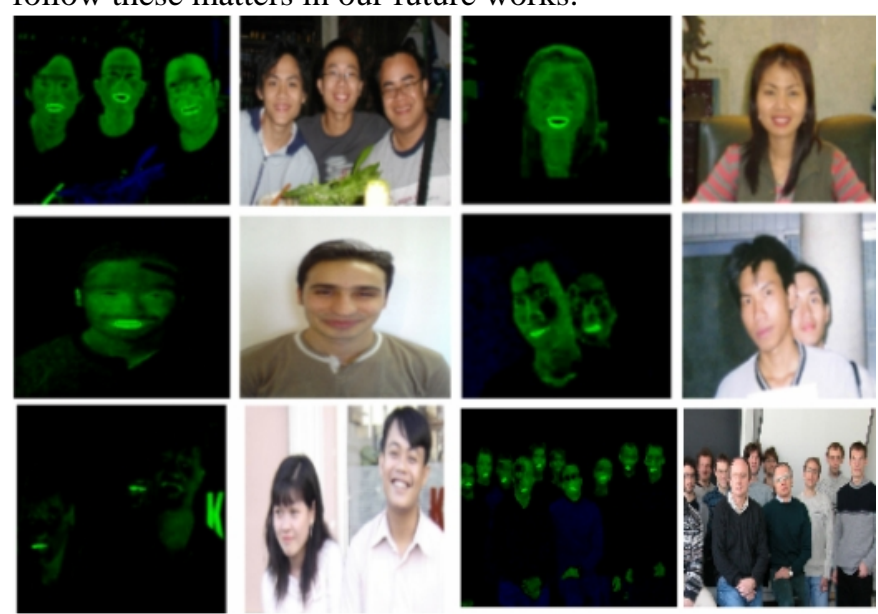

Fig. 4 result of proposed algorithm 


\section{REFRENCES}

[1] Wyszecki, G., Stiles, W.S., 1982. Color Science: Concepts and Methods, Quantitative Data and Formulae, Second Edition Wiley, New York.

[2] Bhaskaran V, Konstantinides K. Image and video compression standards algorithms and architectures. USA: Kluwer Academic Publishers; 1999.

[3] A. Eisner, D. I. A. MacLeod "Blue Sensitive Cones do not Contribute to Luminance, " Journal of the Optical Society of America, vol. 70, pp.121123,1980

[4] R. M. Boynton, R. T. Eskew, C. X. Olson, "Blue Cones Contribute to Border Distinctness, " Vision Research, vol.25, no.9, 1349-1352, 1985

[5] A. Stockman, D. I. A. MacLeod, D. D. DePriest, "The Temporal Properties of the Human Short-Wave Photoreceptors and Their Associated Pathways, " Vision Research, vol.31, 189-209, 1991

[6]I. Philipp, T. Rath, "Improving Plant Discrimination in Image Processing by Use of Different Color Space Transformations, " Computers and Electronics in Agriculture, vol.35, pp.1-15, 2002.

[7] L. Sigal, S. Sclaroff, V. Athitsos, "Skin Color-Based Video Segmentation under Time-Varying Illumination, " IEEE Trans. on Pattern Analysis and Machine Intelligence, vol. 26, no.7, pp.862-877, July 2004.

[8] C. F. Jones, A. L. Abbott, "Optimization of Color Conversion for Face Recognition, " Eurasip Journal on Applied Signal Processing, vol.4, pp.522-529, 2004

[9] A. W-C. Liew, S.H. Leung, W. H. Lau, "Segmentation of Color Lip Images by Spatial Fuzzy Clustering, " IEEE Trans. on Fuzzy System, vol.11, no.4, pp.542-549, Aug. 2003.

[10] A. Diplaros, T. Gevers, I. Patras, "Combining Color and Shape Information for Illumination-Viewpoint Invariant Object Recognition, " IEEE Trans. on Image Processing, vol.15, no. 1, pp.1-11, Jan. 2006.

[11] L. Jin, D. Li, "A Switching Vector Median filter Based on the CIELAB Color Space for Color Image Restoration, " Signal Processing, vol.87, pp.1345-1354, 2007.

[12] C.C. Brunner, A.G. Maristany, D.A. Butler, D. Vanleuween, J.W. Funck, "An Evaluation of Color Spaces for Detecting Defects in Douglas-.r veneer, Ind. Metrol. 2 (3 and 4) (1992) 169-184.

[13] E. Littmann, H. Ritter, "Adaptive Color Segmentation. A Comparison of Neural and Statistical Methods, " IEEE Trans. Neural Networks 8 (1) (1997) 175-185.

[14] W.K. Pratt, Digital Image Processing, Wiley, New York, 1991

[15] P.K. Robertson, Perceptual color spaces, IEEE Comput. Graphics Appl. 9 (1988) 50-64.3189.

[16] L. Yao and W. A. Sethares, "Nonlinear parameter estimation via the genetic algorithm" IEEE Trans. on Signal Processing

First A. Author: Seyyed Meysam Hosseini was born in Firuzkuh, Iran, in 1983. He received the B.S degree in Electronic engineering from Tarbiat moalem University of Sabzevar, Sabzevar, Iran, in 2007. He is now a M.S student in electrical engineering in University of Birjand, Iran. His research interests include image processing and intelligent computing. E-mail:M.Hosseini@Birjand.ac.ir

B. Author: Hasan Farsi was born in Birjand, Iran, in 1968. He received the B.S. and M.s. degree in electrical engineering from sharif University of Technology, iran respectively, and then he received the $\mathrm{PhD}$ degrees in electrical engineering from University of Surey, UK. He works in Engineering Department as an assistant professor at University of Birjand. E-mail:H.Farsi@Birjand.ac.ir

C. Author:Hadi Sadoghi Yazdi was born in Sabzevar, Iran, in 1971. He received the B.S. degree in electrical engineering from Ferdowsi Mashhad University of Iran in 1994, and then he received the M.S. and PhD degrees in electrical engineering from Tarbiat Modarres University of Tehran, Iran, in 1996 and 2005 respectively. He works in Engineering Department as an assistant professor at Tarbiat Moallem University of Sabzevar. His research interests include adaptive filtering, image and video processing, and optimization in signal processing. He has more than 100 journal and conference publications in subject of his interests.

E-mail:sadoghi@sttu.ac.ir 